\title{
THE APPLICATION OF MULTI- DIMENSIONAL RADAR MAPPING MODEL (MDRM-MODEL) ON THE PEOPLE'S REPUBLIC OF CHINA (PRC) AND THE U.S. ECONOMY
}

\author{
Mario Arturo Ruiz Estrada
}

\begin{abstract}
This paper proposes a new multidimensional graphical model to monitoring the GDP growth rates in real prices behavior and the GDP formation accumulation from any country in the short and long run through the uses of "the Multi-Dimensional Radar Mapping Model (MDRM- Model)." Hence, the MDRM-Model is based on the plotting of different coordinates that represents a large number of GDP growth rates in real prices on the top of a large number of perimeters. Each perimeter represents different periods of time in our analysis. In our case, we are using the Multi-Dimensional Radar Mapping coordinate system (MDRM-Coordinate System) to build the MDRM-Model respectively. Moreover, the MDRM-Model can facilitate the visualization of different GDP growth rates in real prices simultaneously in different periods of time (years) or spaces (countries) that follows a logical order into the same graphical space. The MDRM-Model was applied on the economies of People's Republic of China (PRC) and U.S. to analyze the process of GDP formation accumulation in the last past forty years in both economies.
\end{abstract}

Keywords: Econographicology, Economic Modeling, Macroeconomic Policy, Economic Teaching, Multi-Dimensional graphs and Multi-Dimensional Physical Spaces.

\section{An Introduction to the Multi-Dimensional Radar Mapping Model: A Theoretical Framework}

The multi-dimensional radar mapping model is a multi-dimensional coordinate system. The construction of the MDRM-Model is based on the application of the Econographicology framework according to Ruiz Estrada. ${ }^{1}$ Hence, the MultiDimensional Radar Mapping Coordinate System (MDRM-Coordinate System) is formed by a large number of general spaces (axes), at the same time each general space shows a large number of sub-spaces (sub-axes). The center part of the MDRM-Coordinate System is equal to zero. The number zero will be called the epicenter, from this epicenter start to branch out all the general spaces (axes), in our case we like to call to this epicenter "the origin point" that is connected among all the general spaces (axes) together in the same graphical space (see Figure 1: Picture 1). It is important to mention is that each general space (axis) is divided in three sections follow by positive integers, negative integers and 0 . The value is plotted on each general space (axis) can be a positive value, negative value or equal to zero respectively. We can observe that each general space

1 M. A Ruiz Estrada, "Econographicology", International Journal of Economic Research (IJER). Vol. 4, No.1, 2007, pp 93-104. 
(axis) permit the allocation of any value on its coordinate system based on plot the value on its sub-space (sub-axis). The first general space starts from the general space 0 . In the case of the general space 0 has values between 0 and infinity ( $\infty . .$.$) . Hence, all the$ general spaces start to move from the general space 0 until the general space infinity $(\infty) \ldots$ But the value is plotted on its sub-space in the same general space respectively. If we plot each value into its sub-space in the same general space then we can start to join all values are located into its sub-space. Therefore, we can proceed to join value by value from the same general space and its sub-space through strait lines until we can generate a single a large manifold into the same graphical space and time (see Figure 1: Picture 2).

If we analyze different manifolds under different periods of time then we can observe the changes are happen between two or more periods of time. Or we can compare two different phenomena or spaces (countries or regions) behavior from its manifold behavior respectively. If we use our imagination is possible to appear a large and flat manifold with different sizes in each corner and angles into the same graphical space, where each value located on each sub-space in the same general space, it is pending and join to the next value that also is pending on its sub-space in the same general space. Finally, if we arrive to the last sub-space from the same general space that is located in the MDRM-Coordinate System then we join the last sub-space from the same general space in analysis with the general space 0 . Therefore, we can close the circuit and build the flat manifold. The values (sub-spaces) are plotted on each general space can be originated by a growth rate or an absolute value that is pending in some space in different period of time, but when we join different sub-spaces (values) from different general spaces at different periods of time, we can apply the economic real time on the construction of the flat manifold, we can simulate a large manifold is keeping in constant movement to observe the different changes across time and space. ${ }^{2}$ We request also the application of the Omnia-Mobilis assumption on the construction of the manifolds. ${ }^{3}$ It is to generate the freedom of mobility of the values (sub-spaces) from different general spaces into its graphical space respective. Hence, we reduce the uses of the Ceteris Paribus assumption in minimum into our graphical model. In fact, we can observe different scenarios into the manifold such as expansion, contraction and stagnation. However, we are available to compare different periods of time or geographical spaces that can be represented by firms, markets, countries, regions. The main idea is to observe the behavior of economic growth into the same graphical space in different periods of time in constant movement.

2 M. A Ruiz Estrada, "Economic Modeling in Real Time”, FEA-Working Paper No. 2009-11, 2009, pp

1-10.

3 M. A Ruiz Estrada, "Policy modelling: Definition, Classification, and Evaluation", Journal of Policy Modeling, No. 33, Vol. 4, 2011, pp 523-536. 
Figure 1: MDRM-Coordinate System

Picture 1

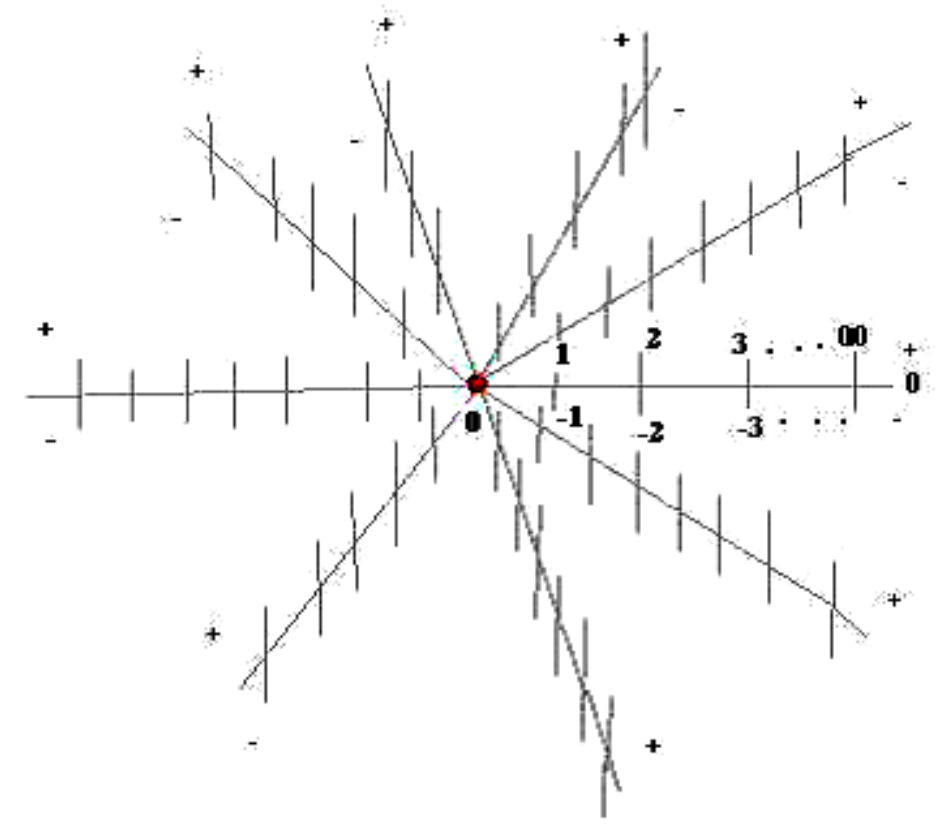

Picture 2

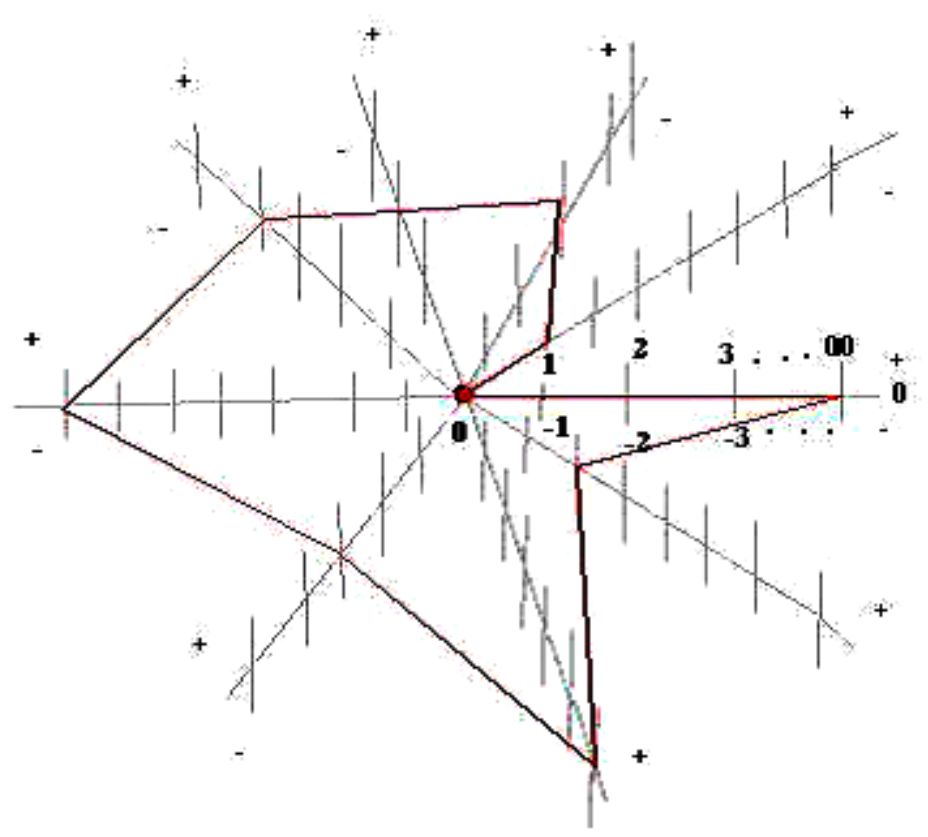




\section{How to Apply MDRM-Model in the Case of PRC and U.S. Economy}

The MDRM-Model can be building under the uses of the MDRM-Coordinate System. In our case we suggest in the construction of the MDRM-Model is based on the uses of a large number of GDP growth rates in real prices from U.S. and People's Republic of China (PRC) performance from 1970 to 2000. We assume that the general space 0 in our MDRM-Model is represented by the GDP growth rates in real prices from year 1970. Both countries shows different GDP growth rate in real prices from year 1970. In the case of the U.S., we can observe a high growth rate located in 4.9\% (NBER, 2014) but in the case of the PRC only arrives to the GDP growth rate in real prices of $1.2 \%(\mathrm{WB}, 2014)$.

The behavior of the GDP growth rates in real prices expansion at U.S. shows a constant expansion with not so much margin or gaps from beginning (see Figure 2: Picture 1), but in the case of the PRC experience a spiral effect (or spiral form) that start with very low growth rates in real prices of its GDP until arrive to year 2000 with a large GDP growth rate in real prices of $11 \%$ (see Figure 2: Picture 2). We can observe a progressive staggering of the GPD growth rates in real prices of PRC until our days. The idea to compare both economies between U.S. and PRC is to study a historical behavior of the GDP growth rates in real prices in both countries from a multi-dimensional perspective and how both economies show different trends and behavior in its GDP growth rates in real prices.

Finally, the MDRM-Model can shows the GDP formation accumulation structure respectively. We can observe in this research that deep impact of externalities, political, social, technological and economic changes can bring a dramatically changes in the behavior of the GDP in real prices and the GDP formation accumulation in both economies simultaneously. The fast expansion of the GDP formation accumulation is depend on the interaction and adaptability of these two economies to fast domestic and international challenges that request efficient allocation of the production factors and a constant monitoring macroeconomic variables to reduce the possibility of a huge damage(s) from possible crisis such as economic crisis, financial crisis, international trade crisis, natural disasters crisis, climate changes crisis, war, crisis. Therefore, we found that the fast expansion of the GDP formation accumulation is result from a deep transformation of the society mind to adapt a fast and deep challenges economically and politically under a strong visionary leadership from the political class in power.

At the same time, the generation of favorable economic and social environments to attract foreign direct investment (FDI) flows in large amounts, free trade agreements, financial and banking strategic alliances with common economic interests from both sides. In addition, the geographical position of any country always is going to play an important role to generate favorable conditions to generate a strong and large GDP formation accumulation for any economy. Finally, the behavior of the GDP formation accumulation between the U.S. and PRC can show clearly that the implementation of a successful economic and political model need to be adapted to its specific economic, social, cultural, technological infrastructure, education, political system characteristics. We cannot implement or import political and economic models such as general and standardized recipe from successful cases such as the PRC to developing countries because each political and economic model need to born and be adapted according to the necessities, limitations and possibilities that any country exist. However, we can remark that the expansion of the GDP formation accumulation of any country 
also depend from the interaction of the civil society, government and private sector together to find common interests such as a better distribution of the income and earnings through better policy modeling approaches specially in the case fiscal policy and alternative social security schemes. We like to remark that the expansion of the GDP formation accumulation is not a simple accumulation of few years, it is a long process with difficulties and vulnerabilities that any country can experience any time. Therefore, the idea to introduce the MDRM-Model is to observe a long historical data of the GDP growth rates in real prices to visualize graphically the evolution of the economic growth from a multi-dimensional perspective.

Figure 2: GDP formation accumulation of U.S. and PRC (1970-2000).

Picture 1: U.S.

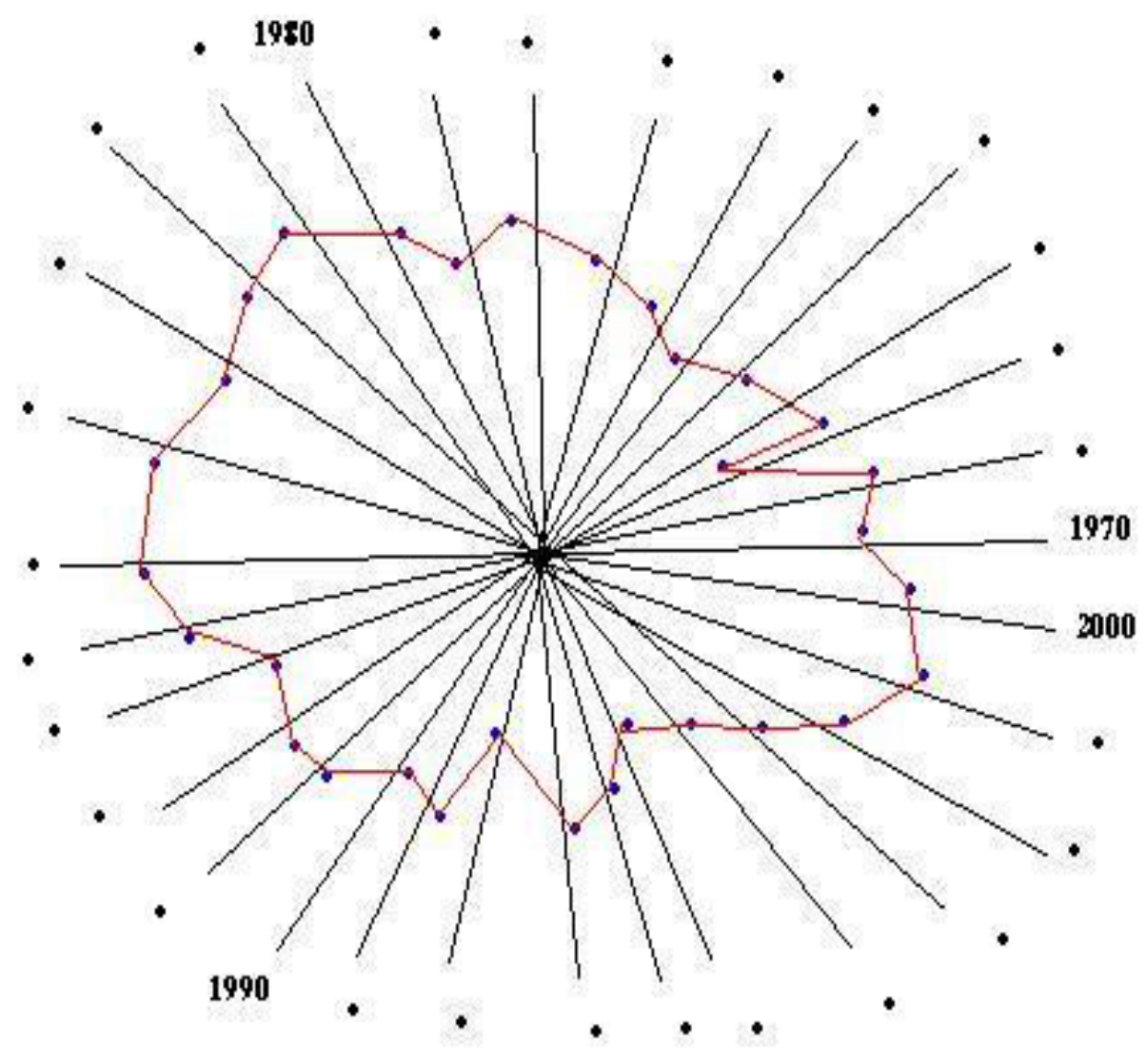


Picture 2: PRC

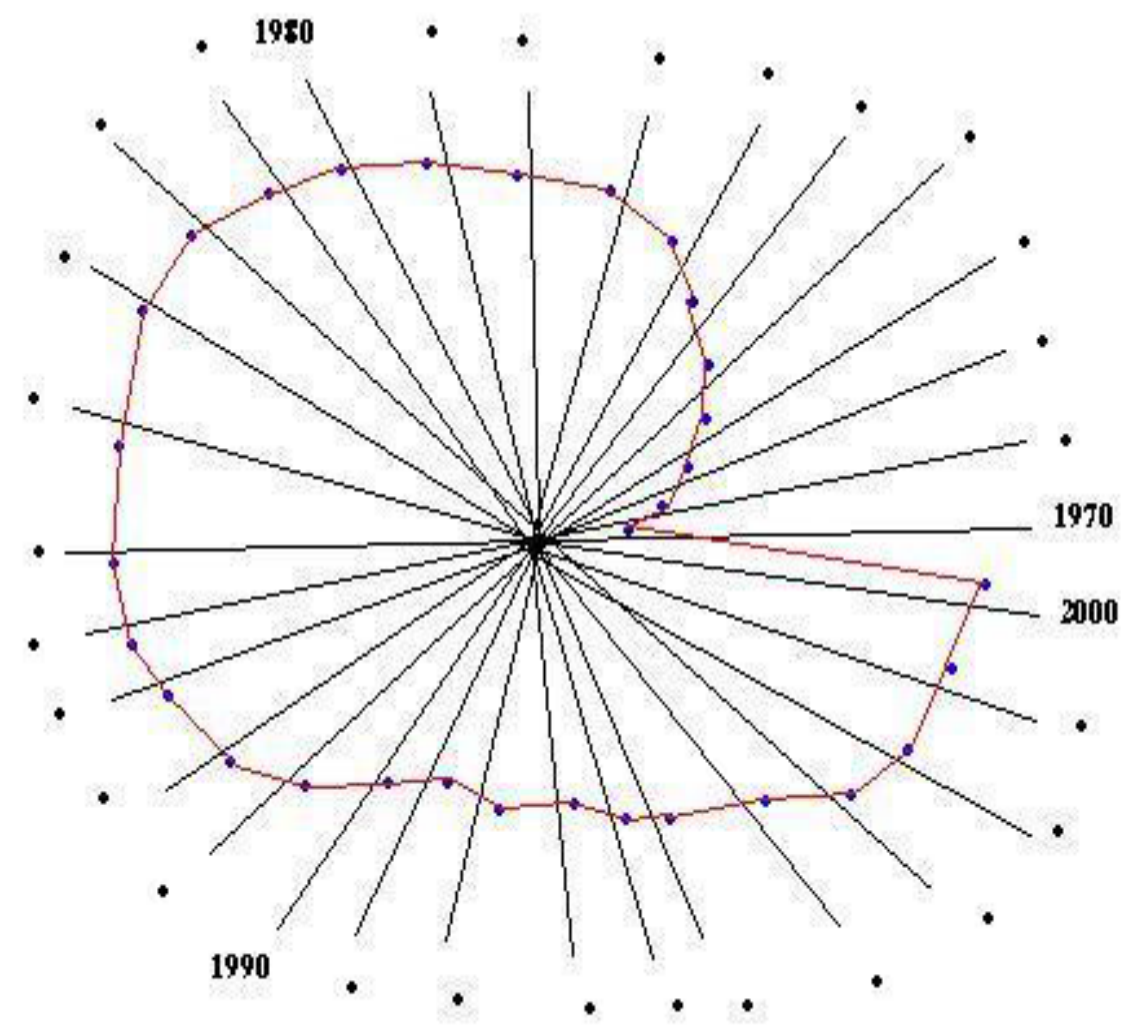

\section{Conclusion}

This paper concludes that the construction of the MDRM-Model can help to us visualize the economic growth in real term of any country from a multi-dimensional perspective that we cannot observe in the classic 2-Dimensional graphical space. The main idea to build the MDRM-Model is to visualize graphically how the evolution and transformation of the U.S. and PRC economy in different periods of time through the application of the Multi-Dimensional Radar Mapping coordinate system (MDRM-Coordinate System). Our conclusion is that the economic growth of U.S. and PRC shows different behavior and react different from internalities and externalities, at the same time, the GDP formation accumulation shows how vulnerable is the U.S. and PRC economy in the short and long run. We can say that these two different economic and political models can show its unique characteristics and different behavior into its process of GDP formation accumulation in the short and long run with different levels of sustainability.

\section{Notes}

Electronic sources were derived from:

National Bureau Economic Research, 2014, available from http://www.nber.org, accessed 10 August 2015.

World Bank General Information and database statistics 2014, available from http://www. worldbank.org, accessed 10 August 2015. 